\title{
MANUAL OPERACIONAL DA ESCALA DE NfVEL REATIVO (RLS 85)
}

\author{
SANDRO ROSSITTI*, JAN-ERIK STARMARK*, DANIEL STALHAMMAR*
}

RESUMO - A Escala de Nivel Reativo (RLS85) é uma escala de coma de oito graus ( scored») pana estimativa do nível de consciência de pacientes com acometimento agudo encefálico. Sua aplicabilidade clínica e em pesquisa foi avaliada em estudo multicêntrico e em estudos de comparação a outras escalas de uso corrente. No presente texto, a manual operacional da RLS85 é sumariado em português, sendo apresentada relação da biblígrafia sobre essa escala.

PALAVRAS-CHAVE: cérebro, acometimento agudo, responsividade, coma, escala, Reaction Level Scale RLS85.

\section{Manual of the Reaction Level Scale (RLS85): a version in Portuguese}

SUMMARY — The Reaction Level Scale (RLS85) is a range scored coma scale (8 levels) for the assessment of responsiveness in patients with acute brain disorders. Its feasibility in clinical pratice and for research studies has been verified in a multicentre study and by comparison with other current coma scales. Range scoring and abscence of pseudoscoring are its most significant adrantages over the other current scales. An operative manual of the RLS85 in Portuguese is standardized in this text which also provides a selected bibliogruphy on the subject. RLS85.

KEY WORDS: brain, acute disorder, responsiveness, coma scale, Reaction Level Scale

A gradação do nível de consciência, ou responsividade, é a parte mais importante do exame clínico de pacientes com afecções encefálicas agudas, tais como traumatismo crânio-encefálico, hemorragias intracranianas não-traumáticas, comas metabólicos e intoxicaçōes. Coma, definido como ausência da consciência de si e do meio na presença de estimulo externo ou de necessidade interna, representa em todos os casos e sem excecão um estado de extrema urgência. O exame clínico nesses casos deve ser simples, objetivo, preciso e confiável. Medidas terapêuticas muitas vezes se sobrepóem ao exame clínico e, frequentemente, profissionais de experiência diversa atuam ao mesmo tempo. Diferentes observadores, sejam médicos ou profissionais paramédicos, devem empregar conceitos semelhantes (padronizados) para a interpretação precisa do nível de consciência e comunicação intra- e interequipes ${ }^{1-3}$. Muitas escalas de coma foram desenvolvidas para esse fim. Numa escala de coma é analisada seleção de aspectos comportamentais, estímulos e reaçōes, sendo negligenciadas as demais va. riáveis. Nenhuma escala substitui, portanto, um detalhado exame clínico e neu. rológico. Algumas escalas têm o inconveniente de serem complexas (e.g. exigindo do observador conhecimento aprofundado dos reflexos segmentais do tronco cerebral) ou de ponderarem variáveis restritas ou inadequadas, às vezes de impossível interpretação (e.g. "abertura dos olhos" quando o paciente apresenta

*Departamento de Neurocirurgia. Universidade de Göteborg, Suécia. Aceite: 12-agosto-1992.

Dr. Sandro Rossitti - Neurokirurgiska kliniken - Sahlgrenska sjukhuset - S-413 45 Göteborg - Sweden. 
pronunciada equimose orbital decorrente de trauma). Mesmo a definição operacional de "coma" varia entre diferentes escalas e os pacientes são agrupados de modo a tornar difícil, ou impossivel, a comparação de diversos estudos $1,5,7,12$. Uma escala de coma ideal deve permitir o reconhecimento de grupos mais ou menos homogêneos de pacientes, permitindo comparar condutas distintas e avaliação estatística do prognóstico para uma mesma condição.

A Escala de Nível Reativo (RLS85 ou "Reaction Level Scale") foi desenvolvida no Departamento de Neurocirurgia da Universidade de Göteborg (Suécia) $3,5,6,9,13$. Essa escala é recomendada pelas sociedades suecas de neurocirurgia e de anestesia para uso geral 8. A RLS85 é escala linear de 8 graus ("níveis reativos") agrupados em 2 categorias mais abrangentes (respectivamente níveis 1-3 e 4-8), delimitadas pelo conceito de "responsividade mental". A atual versão da RIS85 é recomendada para exame na fase aguda (até 3 ou 4 semanas após a lesão) em pacientes com idade minima de 8 anos. Sua confiabilidade, validade e concordância interobservadores foi demonstrada em estudo multicêntrico14, seu valor preditivo tem sido repetidamente comprovado $4,11,15,16$, correlação entre 0 nível reativo e o metabolismo cerebral após trauma severo foi demonstrada 4 e estudos comparativos a outras escalas de uso corrente têm demonstrado que a RLS85 apresenta certas vantagens 10,14,16. Não há pseudo-scores quando se emprega a RLS85 e situações especiais - como pacientes com tubo endotraqueal, equimose orbitária, com deficiências sensoriais ou afásicos - não representam qualquer dificuldade 9 .

A RLS85 foi descrita anteriormente em português, em suas linhas gerais 1 . No presente texto é apresentada padronização operacional da RLS859,13 (Tabela-1).

Tabela 1. Escala de Nivel Reativo (RLS85).

A. Paciente com responsividade mental:

1. Alerta. Resposta imediata.

2. Sonolento ou confuso. Responsivo a estímulo leve.

3. Muito sonolento ou confuso. Responsivo a estímulo intenso.

B. Ausência de responsividade mental:

4. Inconsciente. Localiza mas não afasta estimulo doloroso.

5. Inconsciente. Movimentos de retirada a estímulo doloroso.

6. Inconsciente. Movimento flexor estereotipado a estímulo doloroso.

7. Inconsciente. Movimento extensor estereotipado a estímulo doloroso.

8. Inconsciente. Não responsivo a dor.

\section{MANUAL OPERACIONAL DA RLS85.}

Contato inicial - o termo «reativo» é intencional: c examinador aplica ao paciente estímulos de intensidade progressiva até a definição de seu «nível reativo». Inicialmente conversando em tom de voz normal ou tocando o paciente (para despertá-lo) e, na medida do necessário, elevando o tom de voz e aplicando estímulos físicos de intensidade progressiva, de sacudidas até a estímulos dolorosos. São padronizados dois estímulos dolorosos, que provocam dor de intensidade adequada com mínima lesão tissular: compressão do leito ungueal e compressão digital retromastóidea. Pacientes com suspeita de intoxicação (álcool, medicamentos, narcōticos) apresentam melhor resposta com fricção esternal ou pinçamento digital do músculo trapézio. Importante: estímuios dolorosos aumentam a pressão intracraniana, o que deve ser considerado em função da frequência com que o paciente é examinado.

1. ALERTA. RESPOSTA IMEDIATA.

Alerta: năo sonolento; orientado.

Paciente entubado: nenhuma demora em reagir.

2. SONOLENTO OU CONFUSO. RESPONSIVO A ESTMULo LEVE.

Sonolento: o paciente está sonolento quando assim aparentar demorando a reagir. 
Confuso: o paciente está confuso se cesponder incorretamente a uma das questões: (a) «Qual é seu nome?» (nome completo); (b) «Onde você está?» (local. e.g. nome da cidade ou «no hospital»; (c) Em que mês e ano estamos?».

Estímulo leve: dirigir-se ao paciente verbalmente, ou toca-lo.

\section{MUto sonolento ou confuso. REsponstyo a estimuLo intenso.}

Estímulo intenso: dirigir-se ao paciente em tom alto de voz, sacudi-lo, ou aplicar-lhe estímulo doloroso.

A diferença entre os niveis 2 e 3 baseia-se na intensidade do estímulo.

CONCEITO DE «RESPONSIVIDADE MENTAL» - O paciente é despertável, realizando ao menos uma das seguintes ações: (a) resposta verbal com palavras (ao menos uma única palavra compreensível); (b) movimentos oculares orientados, i.e. abertura dos olhos com contato visual e alguma tentativa de dirigir o olhar; (c) obedece a comandos verbais, e.g. levantar os braços, mostrar a língua, abrir ou cerrar os olhos; (d) localiza e afasta estímulos dolorosos, i.e. o paciente localiza estímulos dolorosos e procura afastá-los ativamente. Observe que: se o paciente não se encontra mentalmente responsivo, porém aparenta estar alerta, devem ser excluídas condiçóes neurológicas e psiquiatricas tais como: estado vegetativo, mutismo acinético, estupor depressivo, estados pré-ictais das epilepsias, histeria, catatonia e sindrome do claustro 3.

\section{INCONSCIENTE. LOCALIZA MAS NAO AFASTA ESTIMULO DoLoRoso.}

Inconsciente: nenhuma responsividade mental. í incapaz de qualquer aça definida anteriormente como responsividade mental.

Localiza a dor: o paciente é examinado em decúbito supino, com os membros superiores pousados ao longo do tronco. À compressão retromandibular o paciente eieva uma das mãos acima do nivel da mandibula. A compressão do leito ungueal em uma das mãos, o paciente movimenta a mão contralateral para além da linha média.

\section{INCONSCIENTE. MOVIMENTOS DE RETIKADA A ESTMULO DOLOROSO.}

Movimentos de retirada: (a) à compressão retromandibular o paciente desvia o rosto para o outro lado; (b) à compressão do leito ungueal o paciente não localiza o estimulo, porém realiza nitidos movimentos de retirada do membro estimulado.

\section{INCONSCIENTE. MOVIMENTO FLEXOR ESTEREOTIPADD A ESTIMULO DOLOROSO.}

Movimento flexor estereotiplado: (a) à compressão retromandibular ou (b) à compressão do leito ungueal, o paciente realiza movimentos flexores do cotovelo e do punho; não se observam movimentos de localização ou retirada. Deve-se anotar sempre a melhor resposta.

\section{INCONSCIENTE. MOVIMENTO EXTENSOR ESTEREOTIPADO A ESTIMULO DOLOROSO.}

Movimento extensor estereotipado: (a) à compressão retromandibular ou (b) à compressão do leito ungueal, obtêm-se movimentos extensores dos membros superiores e inferiores ou, ainda, apenas um reforço tônico da musculatura mímica facial. Nenhum movimento flexor deve ser observado. Deve-se anotar sempre a melhor resposta.

\section{INCONSCIENTE. NAO RESPONSIVO A DOR.}

Nâo responsivo à dor: à estimulação álgica intensa e repetida retromandibular ou do leito ungueal, o paciente não reage com qualquer movimento dos membros ou da face.

Importante - 0 nível reativo 8 não justifica o diagnóstico clínico de morte cerebral.

\section{REFERENCIAS}

1. Balbo RJ, Sperlescu A, Rossitti S. Escalas de coma. Revista Ciências da Saúde 1988, 1:68-70.

2. Lindgren $S$. Diagnostic terminology of head injuries related to severity. In Lindgren $\mathbf{S}$. (ed): Modern concepts in neurotraumatology. Acta Neurochir (Wien) 1986, Suppl 36:70-80.

3. Lindqvist $G$, Malmgren $H$. Organisk psykiatri. Teoretiska och kliniska aspekter. Stockholm: Almqvist \& Wiksell, 1990 p 134-230.

4. Schalén W. Prediction and evaluation of outcome after severe head injury. PhD Thesis, Lunds universitet. Lund, 1992.

5. Starmark JE. Analysing «coma scales». The introduction of a new scale: the Reaction Level Scale (RSL85). PhD Thesis, Göteborgs universitet. Göteborg, 1988.

6. Starmark JE, Carlsson C, Holmgren H, Stalhammar D. Bedömning av medvetande och reaktionsgrad vid traumatiska hjärnskador Läkartidningen 1984, 81:1528-1529. 
7. Starmark JE, Holmgren E, Stalhammar D. Current reporting of responsiveness in acute cerebral disorders. J Neurosurg 1988, 69:692-698.

8. Starmark JE, Stalhammar D. Ny reaktionsgradsskala rekommenderas i Sverige. Läkartidningen 1990, 87:1466-1469.

9. Starmark JE, Stalhammar D, Holmgren E. The Reaction Level Scale (RLS85): manual and guidelines. Acta Neurochir (Wien) 1988, 91:12-20.

10. Starmark JE, Stalhammar D, Holmgren E, Rosander B. A comparison of the Glasgow Coma Scale and the Reaction Coma Scale (RLS85). J Neurosurg 1988, 69:699-706.

11. Starmark JE, Heath A. Severity grading in self-poisoning. Human Toxicol 1988, 7:551-555.

12. Stalhammar D, Starmark JE. Assessment of responsiveness in head injury patients: the Glasgow Coma Scale and some comments on alternative methods. In Lindgren $S$ (ed): Modern concepts in neurotraumatology. Acta Neurochir (Wien) 1986, Suppl 36:91-94.

13. Stalhammar D, Starmark JE. RLS85 handledning: reaktionsgradsskalan. Neurokirurgiska kliniken, Sahlgrenska sjukuset. Göteborg, 1988.

14. Stalhammar D, Starmark JE, Holmgren E, Eriksson N, Nordström CH, Fedders O, Rosander B. Assessment of responsiveness in lacute cerebral disorders: a multicentre study on the Reaction Level Scale (RLS85). Acta Neurochir (Wien) 1988, 90:73-78.

15. Sundbarg G. Neurosurgical intensive care and the management of severe head injuries. PhD Thesis, Lunds universitet. Lund, 1988.

16. Tesseris J, Pantazidis N, Routsi C, Fragoulakis D. A comparative study of the Reaction Level Scale (RLS85) with Glasgow Coma Scale (GCS) and Edinburgh-2 Coma Scale (modified) [E 2 CS(M)]. Acta Neurochir (Wien) 1991, 110:65-76. 